\title{
Correction to: Autonomous Cyber Deception
}

\section{Correction to: \\ E. Al-Shaer et al. (eds.), Autonomous Cyber Deception, https://doi.org/10.1007/978-3-030-02110-8}

This book was inadvertently published as an authored work with the chapter authors mentioned in the footnotes of the chapter opening pages. This has now been updated and the chapter authors have been mentioned in the respective chapter opening pages as mentioned below:

\section{Chapter 1 Using Deep Learning to Generate Relational HoneyData}

Nazmiye Ceren Abay, Cuneyt Gurcan Akcora, Yan Zhou, Murat Kantarcioglu, and Bhavani Thuraisingham

\section{Chapter 2 Towards Intelligent Cyber Deception Systems}

Fabio De Gaspari, Sushil Jajodia, Luigi V. Mancini, and Giulio Pagnotta

The updated online versions of the chapters can be found at https://doi.org/10.1007/978-3-030-02110-8_1 https://doi.org/10.1007/978-3-030-02110-8_2 https://doi.org/10.1007/978-3-030-02110-8_3 https://doi.org/10.1007/978-3-030-02110-8_4 https://doi.org/10.1007/978-3-030-02110-8_5 https://doi.org/10.1007/978-3-030-02110-8_6 https://doi.org/10.1007/978-3-030-02110-8_7 https://doi.org/10.1007/978-3-030-02110-8_8 https://doi.org/10.1007/978-3-030-02110-8_9 https://doi.org/10.1007/978-3-030-02110-8_10 https://doi.org/10.1007/978-3-030-02110-8_11 https://doi.org/10.1007/978-3-030-02110-8 
Chapter 3 Honeypot Deception Tactics

Neil C. Rowe

Chapter 4 Modeling and Analysis of Deception Games Based on Hypergame Theory

Jin-Hee Cho, Mu Zhu, and Munindar Singh

Chapter 5 Dynamic Bayesian Games for Adversarial and Defensive Cyber Deception

Linan Huang and Quanyan Zhu

Chapter 6 CONCEAL: A Strategy Composition for Resilient Cyber Deception: Framework, Metrics, and Deployment

Qi Duan, Ehab Al-Shaer, and Mazharul Islam

Chapter 7 NetShifter: A Comprehensive Multi-Dimensional Network Obfuscation and Deception Solution

Gahng-Seop Ahn, Kyung Joon Kwak, Alexey Bogaevskiy, Jason Li, Gregory Briskin, and Robert Vaeth

Chapter 8 Deception-Enhanced Threat Sensing for Resilient Intrusion Detection

Frederico Araujo, Gbadebo Ayoade, Kevin W. Hamlen, and Latifur Khan

Chapter 9 HONEYSCOPE: IoT Device Protection with Deceptive Network Views

Reham Mohamed, Terrence O'Connor, Markus Miettinen, William Enck, and Ahmad-Reza Sadeghi

Chapter 10 gExtractor: Automated Extraction of Malware Deception Parameters for Autonomous Cyber Deception

Mohammed Noraden Alsaleh, Jinpeng Wei, Ehab Al-Shaer, and Mohiuddin Ahmed

Chapter 11 Malware Deception with Automatic Analysis and Generation of HoneyResource

Zhaoyan Xu, Jialong Zhang, Zhiqiang Lin, and Guofei Gu

Cover corrected to reflect editor information. 